\title{
V379 Cephei: a quadruple system of two binaries ${ }^{\star \star \star \star}$
}

\author{
P. Harmanec ${ }^{1,2}$, P. Mayer ${ }^{1}$, A. Prša ${ }^{3}$, H. Božić ${ }^{4}$, P. Eenens ${ }^{5}$, E. F. Guinan ${ }^{6}$, G. McCook ${ }^{6}$, P. Koubský ${ }^{2}$, \\ D. Ruždjak ${ }^{4}$, S. Engle ${ }^{6}$, D. Sudar ${ }^{4}$, P. Škoda ${ }^{2}$, M. Šlechta ${ }^{2}$, M. Wolf ${ }^{1}$, and S. Yang ${ }^{7}$ \\ 1 Astronomical Institute of the Charles University, Faculty of Mathematics and Physics, V Holešovičkách 2, 18000 Praha 8, \\ Czech Republic \\ e-mail: Petr.Harmanec@mff.cuni.cz \\ 2 Astronomical Institute of the Academy of Sciences, 25165 Ondřejov, Czech Republic \\ 3 Department of Physics, University of Ljubljana, Jadranska 19, 1000 Ljubljana, Slovenia \\ ${ }^{4}$ Hvar Observatory, Faculty of Geodesy, Zagreb University, 10000 Zagreb, Croatia \\ 5 Departamento de Astronomia, Universidad de Guanajuato, Apartado 144, 36000 Guanajuato, GTO, Mexico \\ 6 Department of Astronomy and Astrophysics, Villanova University, 800 Lancester Avenue, Villanova, PE 19085, USA \\ 7 Physics \& Astronomy Department, University of Victoria, PO Box 3055 STN CSC, Victoria, BC, V8W 3P6, Canada
}

Received 29 September 2006 / Accepted 2 November 2006

\begin{abstract}
Context. Several binaries with masses anomalously small for their observed spectral types have been discussed in the astronomical literature and interpreted as the result of envelope ejection. V379 Cep is one of these objects.

Aims. We obtained new series of electronic spectra and $U B V$ photometry of V379 Cep and analysed them in an effort to check whether the conclusion about its anomalous masses may not be premature.

Methods. Reduction of new spectra was carried out in the IRAF and SPEFO programs. New photometry was reduced and transformed to the standard system using the HEC22 program. Orbital elements were derived with the FOTEL program and via disentangling with the program KOREL. The final combined solution was obtained with the program PHOEBE. Some initial period searches were carried out using the phase dispersion minimalization technique.

Results. We found that the second system of spectral lines seen in the spectra of V379 Cep does not belong to the secondary but to the primary of another binary $\mathrm{Ba}+\mathrm{Bb}$ (orbital period of $158.71 \pm 0.17$ ), which constitutes a quadruple system with the narrow-lined $99.7638 \pm 0.0006$ eclipsing binary $\mathrm{Aa}+\mathrm{Ab}$ (for which we report probable detection of the secondary and derive improved period and orbital elements). The mutual motion of the two binaries $\mathrm{A}+\mathrm{B}$ around the common centre of gravity is also observable, and the probable orbital period is $(7878 \pm 77)$ days. Our result shows that the components Aa and Ba are somewhat evolved but removes the reported discrepancy of anomalously small masses.

Conclusions. We find that V379 Cep is an astrophysically interesting quadruple system for which a future combination of interferometry, spectroscopy, and photometry can provide individual masses of all four bodies.
\end{abstract}

Key words. stars: binaries: eclipsing - stars: early-type - stars: individual: V379 Cep - stars: binaries: spectroscopic stars: fundamental parameters

\section{Introduction}

V379 Cep (HR 7940; HD 197770) is a bright reddened star $(V=6.31, B-V=0.338, U-B=-0.48)$ of spectral type B2 IV-III (Hiltner 1956; Gordon et al. 1998). Searching for new $\beta$ Cep variables, Lynds (1959) was unable to find any rapid variations for this star. For several decades, V379 Cep had been used as the comparison star and a transformation standard in the Be-star observing campaign (Harmanec et al. 1994) until Jerzykiewicz (1993) discovered that the object is an eclipsing binary with about 0 . 05 deep eclipses in Strömgren $b$. He tentatively suggested a 24.45 period. In 1993-1995 Clayton (1996) reobserved the star in $U B V$ and confirmed its eclipsing binary nature. Combining his observations with those by Jerzykiewicz (1993) he found that the correct orbital period is $99.76 \pm 0.04$

* Based on new spectral and photometric observations from the following observatories: Dominion Astrophysical Observatory, Hvar, Ondřejov, Fairborn and San Pedro Mártir.

$\star \star$ Table 2 is only available in electronic form at the CDS via anonymous ftp to cdsarc.u-strasbg.fr $(130.79 .128 .5)$ or via http://cdsweb.u-strasbg.fr/cgi-bin/qcat?J/A+A/463/1061 and that the primary and secondary minima of similar depths are separated by just one half of the orbital period. Variable radial velocity (RV hereafter) had already been observed by Adams et al. (1924). Gordon et al. (1998), who analysed electronic spectra obtained between 1992 and 1997 at two observatories, found RV variations with a period of $99.69 \pm 0.02$, and demonstrated that two sets of spectral lines are present, a broad one with $v \sin i=55 \mathrm{~km} \mathrm{~s}^{-1}$ and a narrow one with $v \sin i=15 \mathrm{~km} \mathrm{~s}^{-1}$. Identifying them with the primary and secondary components of a binary system, they derived an eccentric orbit with $e=0.147$, $\omega=70^{\circ}, M_{1} \sin ^{3} i=2.89 M_{\odot}$, and $M_{2} \sin ^{3} i=1.92 M_{\odot}$. Since the binary eclipses are observed, this implies an orbital inclination not very far from $90^{\circ}$ and, therefore, anomalously low masses for the two stars that - according to them - have both spectral class B2 III. They suggested that V379 Cep consists of highly evolved stars and argued that this is also evidenced by the presence of two shells centred on the stars that are seen in its IRAS IR images. We note, however, that the eccentric orbit with $\omega=70^{\circ}$ published by Gordon et al. (1998) obviously disagrees with the observed primary and secondary photometric eclipses, which are separated by almost exactly a half period. 
Table 1. Journal of available photometric observations of V379 Cep.

\begin{tabular}{ccrccl}
\hline \hline Station* & $\begin{array}{c}\text { Time interval } \\
\text { (HJD-2400 000) }\end{array}$ & $\begin{array}{r}\text { No. of } \\
\text { obs. }\end{array}$ & Passbands & $\begin{array}{c}\text { HD of comparison } \\
\text { /check star }\end{array}$ & Source \\
\hline 05 & $41580.7-41971.6$ & 43 & $b$ & $198679 / 197618$ & Jerzykiewicz (1993) \\
01 & $44431.5-51426.3$ & 176 & $U B V$ & $194668 /-$ & Pavlovski et al. (1997); Harmanec et al. (1997) \\
61 & $47863.9-49046.1$ & 112 & $V$ & - & Perryman \& ESA (1997) \\
15 & $49122.0-50054.6$ & 156 & $U B V$ & $198793 / 199661$ & Clayton (1996) \\
30 & $52064.9-52384.0$ & 17 & $U B V$ & $194668 / 197618$ & this paper \\
01 & $52494.5-53694.2$ & 46 & $U B V$ & $194668 / 197618$ & this paper \\
16 & $53672.6-53714.6$ & 246 & $U B V$ & $194668 / 198793$ & this paper \\
\hline
\end{tabular}

* In column "Station", individual observing stations are distinguished by the running numbers they have in the Praha/Ondřejov photometric archives: 01... Hvar 0.65-m reflector, EMI tube; 05... Lowell Obs. 1.07-m reflector, EMI 6256S tube; 15... Phoenix-10 (0.254-m) Automatic Photoelectric Telescope and photon-counting photometer; 16... Villanova University Four-College 0.8-m Automatic Photoelectric Telescope; 30... San Pedro Mártir 0.84-m reflector and Cuenta-pulsos photometer; 61... Hipparcos all-sky $H_{\mathrm{p}}$ photometry transformed into Johnson $V$.

Eggleton $(2002 \mathrm{a}, \mathrm{b})$ attempted to explain the evolutionary stage of V379 Cep theoretically as the result of envelope ejection.

To shed more light on this intriguing system, we have accumulated new spectral and photometric observations from several observatories. Their interpretation indeed led to a picture that is quite different from the results by Gordon et al. (1998). Our results are reported in the rest of this study.

\section{Observations and reductions}

\subsection{Photometry}

A journal of available photoelectric observations of V379 Cep is given in Table 1, together with information on the comparison and check stars used by different observers. All individual observations are in Table $2^{1}$. Hvar $U B V$ observations secured before 2000 have already been published in detail (Pavlovski et al. 1997; Harmanec et al. 1997). All Hvar, SPM, and Four-College APT $U B V$ observations were carefully transformed into the standard Johnson system via non-linear tranformation formulae with the help of the HEC22 program (rel. 15) (Harmanec et al. 1994). Earlier Hvar observations in which V379 Cep served as the comparison were reduced again with respect to the (at that time check) star HR 7815². The all-sky Hipparcos $H_{\mathrm{p}}$ observations were transformed to Johnson $V$ magnitude using the tranformation derived by Harmanec (1998). Individual $b$ and $U B V$ observations secured by Jerzykiewicz (1993) and Clayton (1996), respectively, were kindly put at our disposal by their authors.

The $U B V$ observations secured by Clayton (1996) have been corrected for differential extinction and transformed to the $U B V$ system via linear transformation formulae by their author. To get them on a scale close to the standardized Hvar, SPM and Four-College APT data, we also observed his comparison star HD 198793 at Hvar and with the 0.8-m APT and added our standard all-sky $U B V$ magnitudes to Clayton's magnitude differences V379 Cep - HD 198793. The standard $U B V$ values of all comparisons used are summarised in Table 3.

\subsection{Spectroscopy}

A journal of our new CCD spectra is in Table 4. They come from three sources:

1. HEROS spectrograph (Kaufer 1988), loaned by the Landessternwarte, Heidelberg, attached to the $2.0-\mathrm{m}$

\footnotetext{
1 Table 2 is only published in electronic form at the CDS.

2 This was feasible thanks to our routine practice observing the check star as frequently as the variable itself.
}

Table 3. Standard $U B V$ magnitudes of the stars used by different observers as comparison stars in their differential observations of V379 Cep. The $U B V$ magnitudes of these stars are based on numerous all-sky observations secured at Hvar.

\begin{tabular}{rcccc}
\hline Star & HD & $\begin{array}{c}V \\
\text { (mag.) }\end{array}$ & $\begin{array}{c}B-V \\
\text { (mag.) }\end{array}$ & $\begin{array}{c}U-B \\
\text { (mag.) }\end{array}$ \\
\hline HR 7815 & 194668 & 6.509 & -0.019 & -0.057 \\
HD 198679 & 198679 & 6.893 & -0.057 & -0.168 \\
HD 198793 & 198793 & 7.128 & -0.076 & -0.476 \\
\hline
\end{tabular}

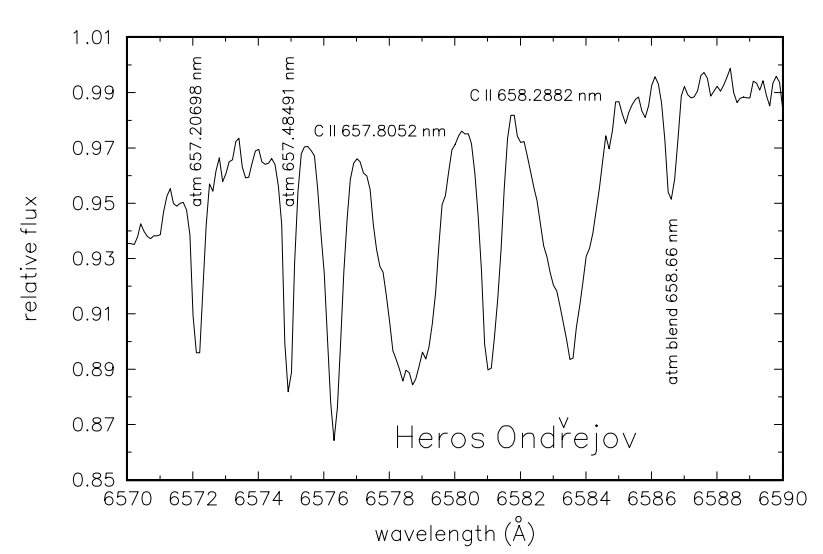

Fig. 1. One Ondřejov Heros spectrum of V379 Cep in the neighbourhood of the C II 6578 and $6582 \AA$ doublet on which the narrow and broader lines are best separated because of large differences in their RVs.

Ondřejov Zeiss Jena reflector; the region covered simultaneously in two passbands is 3600 to $8300 \AA$, linear dispersion $3.9 \AA \mathrm{mm}^{-1}$ at $4000 \AA$, to $6.8 \AA \mathrm{mm}^{-1}$ at $\mathrm{H} \alpha$. The spectral resolution is 20000 over the whole covered range of wavelengths. The $S / N$ of these spectra ranges from 122 to 228 .

2. Coudé spectrograph of the same $2.0-\mathrm{m}$ Ondřejov reflector, equipped with a SITe-005 $800 \times 2000 \mathrm{CCD}$; the spectra cover the region from 6280 to $6720 \AA$, with a linear dispersion of $17 \AA \mathrm{mm}^{-1}$, two-pixel resolution of 12700 , and $S / N$ ranging from 225 to 368 (one underexposed spectrum having 33 only).

3. Coudé spectrograph of the DAO McKellar 1.22-m reflector with a SITe-4 $4096 \times 2048 \mathrm{CCD}$; the spectra cover the region 6200 to $6750 \AA$, with a linear dispersion $10 \AA \mathrm{mm}^{-1}$ and a 
Table 4. Journal of available spectral observations and RV measurements of V379 Cep.

\begin{tabular}{ccrl}
\hline \hline Obs.* $^{*}$ & $\begin{array}{c}\text { Time interval } \\
\text { (HJD-2 400 000) }\end{array}$ & $\begin{array}{r}\text { No. of } \\
\text { RVs. }\end{array}$ & Source \\
\hline 1 & $19560.0-19735.6$ & 4 & Abt (1973) \\
2 & $24039.8-24822.7$ & 8 & Plaskett \& Pearce (1931) \\
3 & $48780.8-48904.6$ & 4 & Gordon et al. (1998) \\
4 & $50345.7-50727.6$ & 17 & Gordon et al. (1998) \\
5 & $52362.5-52490.5$ & 7 & this paper \\
6 & $53335.4-53920.4$ & 18 & this paper \\
7 & $53568.9-53912.9$ & 26 & this paper \\
\hline
\end{tabular}

* In column "Obs.", individual instruments are identified by numbers: 1... Mt. Wilson 1.52-m reflector, 0.46-m camera, single-prism spg.; 2... Dominion Astrophysical Observatory 1.83-m reflector, IM and IS spectra; 3... Kitt Peak National Observatory 0.9-m Coudé feed telescope, echelle CCD spg.; 4... Ritter Observatory 1-m reflector, fiber-fed echelle CCD spg.; 5 ... Ondřejov 2-m telescope, fiber-fed Heros echelle CCD spg.; 6... Ondřejov 2-m telescope, Coudé CCD spg.; 7... Dominion Astrophysical Observatory 1.83-m reflector, Cassegrain CCD spg.

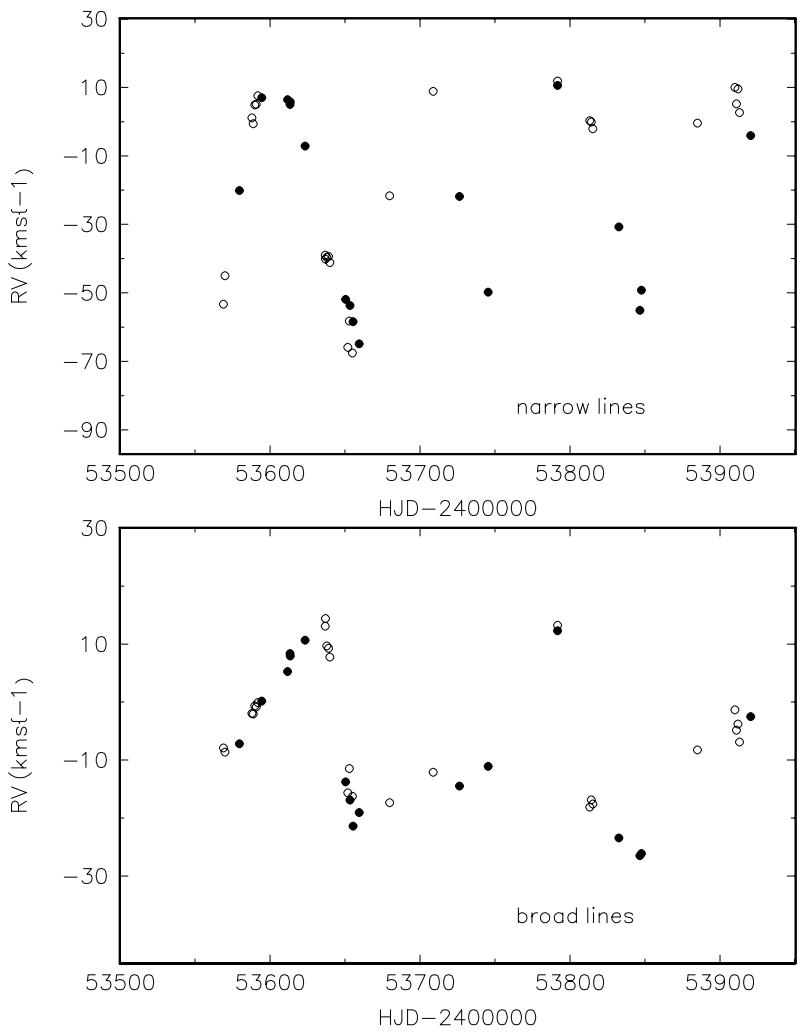

Fig. 2. RV of the narrow and broad lines plotted vs. time for the recent Ondřejov (filled circles) and DAO (open circles) spectra.

two-pixel resolution of 18000 . Their $S / N$ ranges from 93 to 324.

In all cases, the wavelength calibration was based on ThAr comparison spectra taken before and after the stellar exposure. Mean flatfield images from the same night as the stellar spectrum were always applied.

The initial reductions to 1-D frames were carried out in IRAF, by SY for the DAO and by PŠ and MŠ for the Ondřejov spectra - see Škoda \& Šlechta (2002) for details of the Heros spectra reductions. The final reduction, rectification, RV and line-intensity measurements were carried out in SPEFO (Horn et al. 1996; Škoda 1996) by PH. The RV's were measured interactively, comparing the direct and flipped images of the line profiles. The zero point of RV scale was corrected through the use of reliable telluric lines - see Horn et al. (1996) for details.

An example of well-separated narrow and broad lines of C II is shown in Fig. 1. For the final RVs of the sharp lines, we used the mean of the C II 6578.052 and $6582.882 \AA$ doublet, He I $6678.151 \AA$ and $\mathrm{H} \alpha$. For the RVs of the broad lines, we adopted the mean RV of the C II 6578.052 and $6582.882 \AA$ doublet and He I $6678.151 \AA$ only, the $\mathrm{H} \alpha$ being poorly resolved in most cases.

We also compiled all RVs available in the astronomical literature and used the HEC19 program (written by $\mathrm{PH}$ ) to derive the heliocentric Julian dates (HJDs hereafter) for them. In particular, we used the early photographic radial velocities from Mt. Wilson obtained by Adams et al. (1924) and published by Abt (1973) and from the DAO (Plaskett \& Pearce 1931).

\section{Initial analysis of RVs}

All trial solutions of the RV and light curves presented in this study were derived with the FOTEL program (Hadrava 1990, $2004 \mathrm{a}$ ). Because a rather accurate value of the 99.7 orbital period is required for the analyses of the RVs, we initially inspected photometry at phases around both minima. Two early observations from the Hvar Observatory (Oct. 8, 1988: JD 2447443) confirm the phases of minima, and the photometry obtained in July and November 2005 defines the shape of the secondary minimum. Using all available photometry, we derived the following preliminary new ephemeris

$T_{\text {Min.I }}=$ HJD $2441856.6+99.762 \times E$,

which agrees well with the photometric ephemeris derived previously by Clayton (1996). Note that the very narrow minima allow to derive the period with a high accuracy, certainly higher than it is possible from existing spectroscopy. The significantly different period of 99 d.69 used by Gordon et al. (1998) is therefore not supported. The primary minimum is slightly deeper $(0.047$, in $V)$ than the secondary one $(0.035)$. In the preliminary analysis of the RVs described below we therefore kept the period from ephemeris (1) fixed.

When we attempted to plot the RVs of the narrow and broad lines vs. phase of the $99 \mathrm{~d} 762$ period, we found that the RV of the broad lines did not follow a curve in antiphase to those of the narrow-lined star as expected for the secondary star. After we accumulated sufficiently long series of spectra, the reason became apparent: the broad lines belong to the brighter component of another binary with a period of about 159 days. This is illustrated well by Fig. 2 where the RVs from recent DAO and Ondřejov spectra are plotted vs. time. It was easy to verify that RVs of the broad lines published by Gordon et al. (1998) can also be reconciled with a $159-\mathrm{d}$ period. At the same time, it was clear that the systemic velocity of both orbits varies on a time scale of years. This fact can be noted even in the time plots in Fig. 2. To make the conclusion more quantitative, we proceed as follows. We sorted all Gordon et al. (1998) and our new RVs for the narrow and broad lines in time and allowed determination of different systemic velocities for individual data subsets spanning no more than some 400 days. We then derived two trial orbital solutions: for the narrow lines and a period of 99.762, and for the broad lines and a period of 159 d. In Fig. 3 we plot the locally derived systemic velocities from both solutions vs. time. 


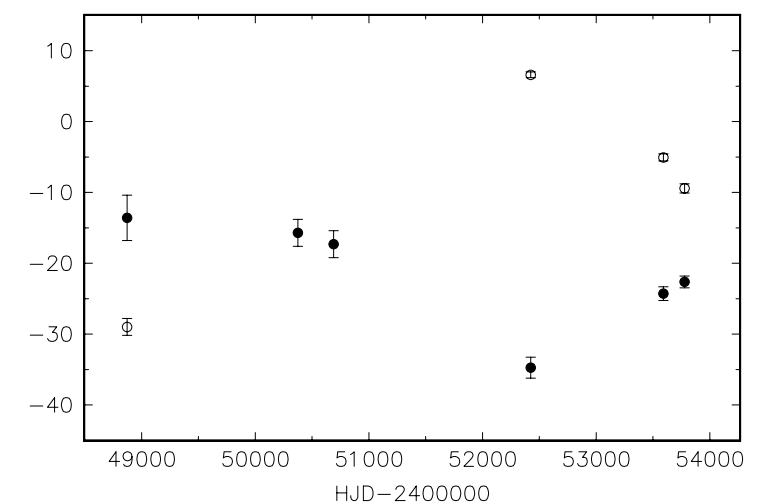

Fig. 3. Locally derived systemic velocities for the narrow lines and a period of 99 d 762 (black dots) and for the broad lines and a period of $159 \mathrm{~d}$ (open circles) plotted vs. time for the RVs from Gordon et al. (1998), Ondřejov and DAO spectra. The rms errors of locally derived systemic velocities for both orbits are shown by bars.

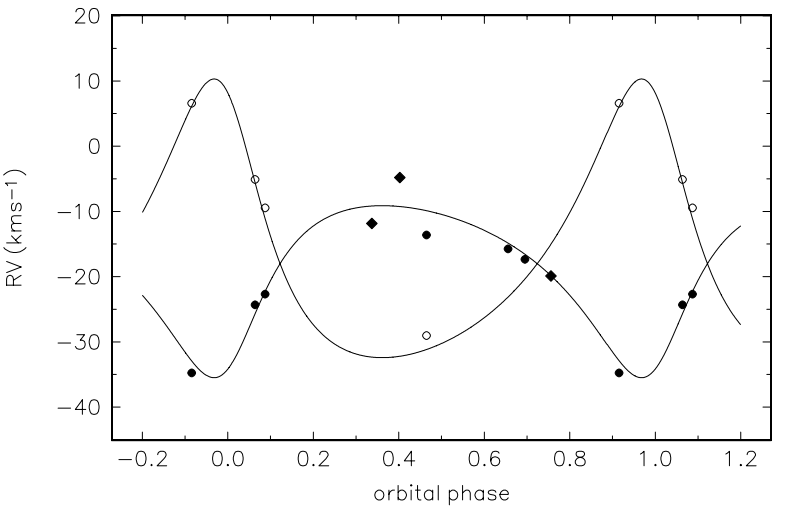

Fig. 4. A phase diagram for the wide orbit with a period of 7878 days as defined by the locally derived systemic velocities for both 99.762 and 159-d binaries. The values from the old data are shown by diamonds, those for the new ones by circles (black ones for the 99.76 binary and empty ones for the 159-d binary).

It is obvious that the two binaries (with the 99.762 and $159 \mathrm{~d}$ orbital periods) move in antiphase around a common centre of gravity with a probable period of some 8000 days. The shape of the curves also indicates that the large orbit has appreciable eccentricity. Hereafter, we denote the primary and secondary of the 99.762 binary as components $\mathrm{Aa}$ and $\mathrm{Ab}$ and the primary and secondary of the 159-d orbit as components $\mathrm{Ba}$ and $\mathrm{Bb}$.

To see whether the old Mt. Wilson and DAO RVs could be used to further improve the period of the large orbit, we derived other trial solutions for these RVs, split again into three subsets for which the systemic RVs could be derived separately. We found that the old RV measurements clearly refer to the narrow system of lines, although with a reduced amplitude ${ }^{3}$. In particular, the epochs of the periastron passage for the 99.76 orbit and the old and more recent RVs were

HJD $2422153.6 \pm 2.8$ and HJD $2453079.68 \pm 0.35$;

these two epochs are separated by 310 full orbital cycles and imply the orbital period of $99.762 \pm 0.008$.

3 The only large deviation was found for a DAO spectrum taken on HJD 2424444.7237 , which has RV of $+17.4 \mathrm{~km} \mathrm{~s}^{-1}$. This could be due to blending with the broad component, and we simply omitted this single RV from our analysis.
Table 5. A FOTEL solution for the locally $\gamma$ velocities for both A and B systems.

\begin{tabular}{cc}
\hline \hline Element & Value \\
\hline$P(\mathrm{~d})$ & $7878 \pm 77$ \\
$T_{\text {periastr. }}$ & HJD $2453090 \pm 260$ \\
$e$ & $0.36 \pm 0.17$ \\
$\omega\left(^{\circ}\right)$ & $206 \pm 15$ \\
$K_{\mathrm{A}}\left(\mathrm{km} \mathrm{s}^{-1}\right)$ & $13.2 \pm 2.9$ \\
$K_{\mathrm{B}}\left(\mathrm{km} \mathrm{s}^{-1}\right)$ & $21.4 \pm 2.0$ \\
\hline
\end{tabular}

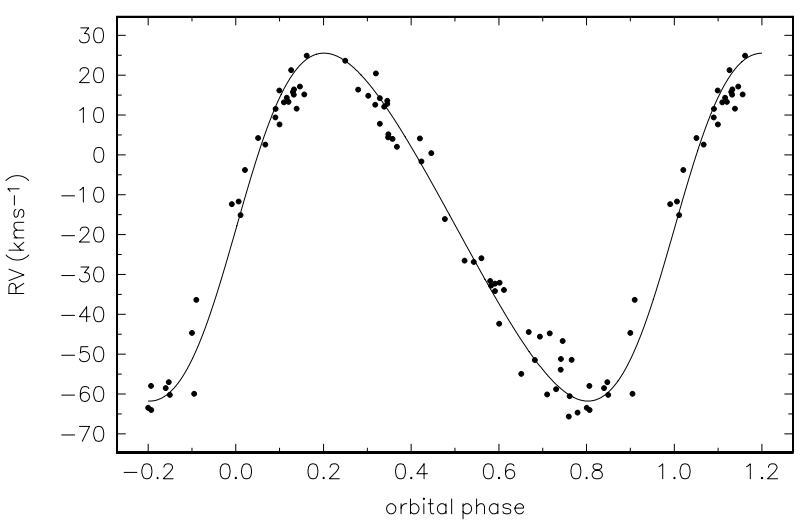

Fig. 5. A phase diagram for the 99.762 orbit and all new RVs based on a FOTEL triple-star solution, i.e. prewhitened for the 7878-d period.

Also adopting the three locally derived $\gamma$ velocities for the old RVs, we derived a trial solution for the $\gamma$ 's from the sharpline system as a "primary" and for the broad-line one as the "secondary". This gave a period of $7878 \pm 77$ days for the large orbit and elements that are summarized in Table 5. The corresponding phase diagram is shown in Fig. 4. We warn that the exact value of the large period depends critically on the early RVs, and continuing observations are certainly very desirable. For further analyses, we keep all elements of the large orbit fixed at the values given in Table 5.

The FOTEL program allows us to treat a triple system, so we used this option to derive orbital RV curves prewhitened for the large orbit. We derived two triple-system orbital solutions. In the first one, we used all the available more recent RVs of component Aa assuming one joint $\gamma$ velocity for them and keeping the orbital periods of 99.762 and $7878 \mathrm{~d}$ and all other elements of the large orbit fixed. We also fixed $\omega=268^{\circ} 3$ for the 99.762 orbit since it is dictated by the shape of the light curve (see below). The corresponding phase diagram for the Aa component, based on RVs prewhitened for the long orbit, is shown in Fig. 5.

The second triple-star FOTEL solution was derived for all recent RVs for the broader system of lines (with the exception of the very first RV from the KPNO, which gives a very large residual), and it gave a period of $159.14 \pm 0.15$ for the B binary, eccentricity of $0.504 \pm 0.026$, periastron epoch HJD $2453002.7 \pm 1.3$, longitude of periastron of $57.1^{\circ} \pm 4.4^{\circ}$, and semiamplitude $K_{\mathrm{Ba}}=17.62 \pm 0.60 \mathrm{~km} \mathrm{~s}^{-1}$. The corresponding phase diagram for the Ba component, based on its RVs prewhitened for the long orbit, is shown in Fig. 6.

\section{Spectral disentangling}

To obtain the best possible orbital elements for all three orbits, disentangling of spectra is preferred to the classical 


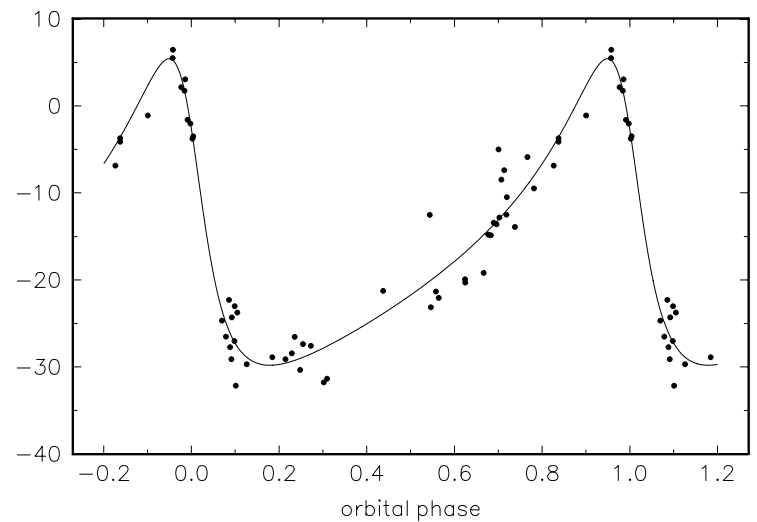

Fig. 6. A phase diagram for the 159-day orbit and all new RVs based on a FOTEL triple-star solution, i.e. prewhitened for the 7878-d period.

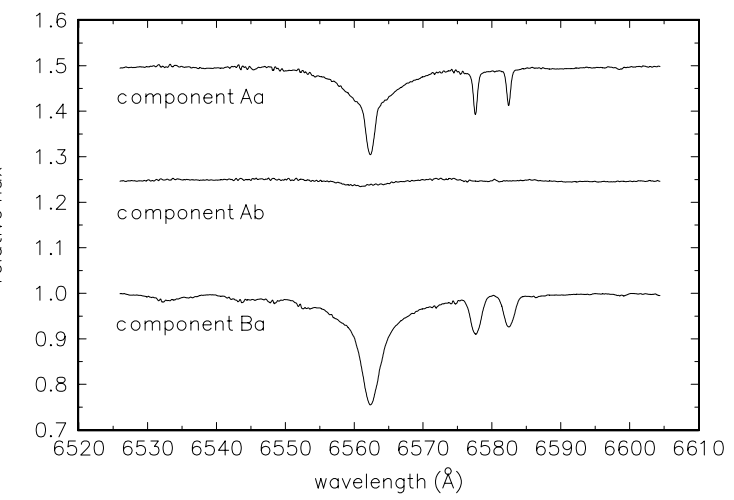

Fig. 7. Profiles of the $\mathrm{H} \alpha$ line and the $\mathrm{C}$ II doublet disentangled in KOREL.

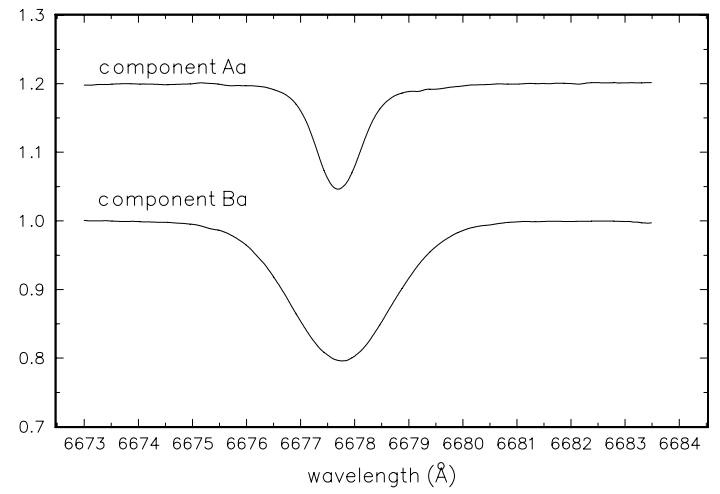

Fig. 8. Profiles of the He I 6678 A line disentangled in KOREL.

RV measurements. We used the KOREL program (Hadrava 1995, 1997, 2004b, 2005), which is particularly suited to our problem since it allows a simultaneous solution for a hierarchical system of five stars.

We first measured the $S / N$ for all spectra and weighted them with weights proportional to $(S / N)^{2}$ and normalized to one for the mean value. These weights were used in the control file of the auxiliary program PREKOR (Hadrava 2004b) that prepares the input data for KOREL. Following the advice of the author of the program, we first derived the line strengths of the telluric spectra for the wavelength range 6465-6510 $\AA$, which contains a number of strong water vapour lines and then kept these strengths fixed in all subsequent solutions with KOREL.

Another complication that must be kept in mind is that the spectrum of V379 Cep contains several quite strong interstellar

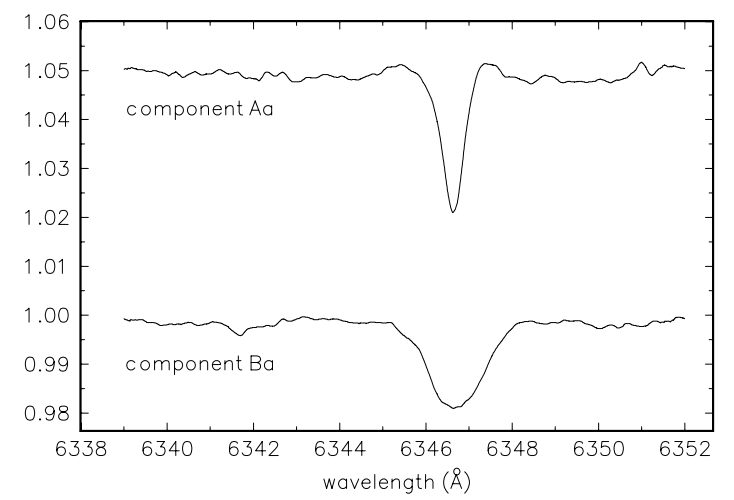

Fig. 9. Profiles of the Si II $6347 \AA$ disentangled in KOREL.

Table 6. KOREL solutions for the quadruple system for three spectral regions, with all epochs in HJD-2 400000.

\begin{tabular}{cccc}
\hline \hline Element & $\mathrm{H} \alpha$ & He I 6678 & Si II \\
\hline System Aa $+\mathrm{Ab}$ & & & \\
$P(\mathrm{~d})$ & 99.7623 fixed & 99.7623 fixed & 99.7623 fixed \\
$T_{\text {periastr. }}$ & 53079.883 & 53079.791 & 53079.351 \\
$e$ & 0.143 & 0.122 & 0.109 \\
$\omega\left({ }^{\circ}\right)$ & 268.3 fixed & 268.3 fixed & 268.3 fixed \\
$K_{1}\left(\mathrm{~km} \mathrm{~s}^{-1}\right)$ & 43.4 & 44.4 & 42.4 \\
$K_{2}\left(\mathrm{~km} \mathrm{~s}^{-1}\right)$ & 75.7 & - & - \\
\hline System Ba $+\mathrm{Bb}$ & & & \\
$P(\mathrm{~d})$ & 158.95 & 158.54 & 158.64 \\
$T_{\text {periastr. }}$ & 53004.120 & 53004.550 & 53004.775 \\
$e$ & 0.504 & 0.493 & 0.530 \\
$\omega\left({ }^{\circ}\right)$ & 57.2 & 59.3 & 61.7 \\
$K_{1}\left(\mathrm{~km} \mathrm{~s}^{-1}\right)$ & 17.9 & 18.1 & 16.5 \\
\hline System A $+\mathrm{B}$ & & & \\
$P(\mathrm{~d})$ & 7878 fixed & 7878 fixed & 7878 fixed \\
$T_{\text {periastr. }}$ & 53091 fixed & 53091 fixed & 53091 fixed \\
$e$ & 0.36 fixed & 0.36 fixed & 0.36 fixed \\
$\omega\left({ }^{\circ}\right)$ & 206 fixed & 206 fixed & 206 fixed \\
$K_{1}\left(\mathrm{~km} \mathrm{~s}^{-1}\right)$ & 12.8 & 12.7 fixed & 13.7 \\
$K_{2}\left(\mathrm{~km} \mathrm{~s}^{-1}\right)$ & 21.2 & 20.5 fixed & 18.4 \\
\hline & & &
\end{tabular}

lines. The parts of spectra containing these lines must be avoided. For this reason, we derived the KOREL solutions separately for the following three regions: 6339-6352 ^ containing the Si II $6347 \AA$ line, 6526-6604 $\AA$ containing the H $\alpha$ and C II lines, and 6673-6683 A containing the He I $6678 \AA$ line.

KOREL was able to disentangle a very weak $\mathrm{H} \alpha$ line of the component $\mathrm{Ab}$, although we admit that this detection is still tentative and requires confirmation with a larger number of spectra. Disentangled spectra near $\mathrm{H} \alpha$, He I 6678, and around Si II $6347 \AA$ line are shown in Figs. 7-9. The orbital elements derived by KOREL for all three regions are summarised in Table 6. Note that the available versions of KOREL do not provide the errors of the elements, therefore the comparison of the results for the three regions gives some idea about the actual accuracy of the derived elements. Note also that disentangling cannot provide information on the systemic velocity. Its value can only be obtained by a direct measurement of disentangled line profiles. This way, we found that the systemic velocity of the whole quadruple system is about $-20 \mathrm{~km} \mathrm{~s}^{-1}$. 


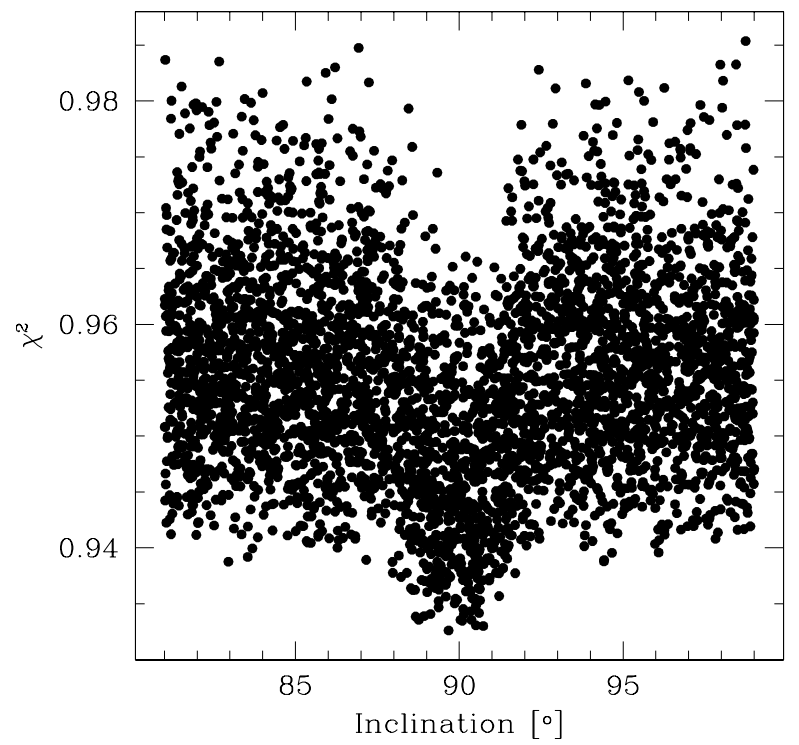

Fig. 10. An example of the results of Monte Carlo search for the optimal orbital parameters: the distribution of $\chi^{2}$ values projected to the inclination. The term projection is used to indicate that the inclination was not the only parameter that was being adjusted, hence the dispersion of $\chi^{2}$ values along the same values of inclination. The result clearly indicates that its value is derived very reliably.

\section{Combined light-curve and orbital solution for the $\mathrm{Aa}+\mathrm{Ab}$ binary}

For full-fledged modelling of all available data we used the PHOEBE program version 0.30 (Prša \& Zwitter 2005) with the WD back-end (Wilson \& Devinney 1971). To improve the $S / N$ of photometric data, we created 0.06 normal points (the typical length of Hvar observations of one group) and removed data points for which the rms errors exceeded 0.01 and a few other deviating points. We excluded all incomplete $U B V$ observations from the Phoenix APT, since the fact that not all three filters were obtained usually indicates either a technical problem or bad weather. RVs for the final solutions were taken from the KOREL solution. For component Aa, mean RVs from three separate KOREL solutions for the $\mathrm{H} \alpha$, He I 6678, and Si II regions were adopted, while for component $\mathrm{Ab}$ only $\mathrm{H} \alpha \mathrm{RV}$ was used.

Below we give a quick overview of the most important modelling steps. To establish a reliable estimate of orbital parameters (semi-major axis $a$, inclination $i$, eccentricity $e$, and argument of periastron $\omega$ ), we fitted the model to photometric and RV data simultaneously. Since there are still only a few data points in the eclipses, the standard minimizers did not converge reliably. For that reason we resorted to a Monte Carlo simulation with 10000 random trial combinations of the afore-mentioned parameters. In addition, a conditional constraint between the semimajor axis and the inclination, $a \sin i=231 R_{\odot}$, as determined by the radial velocity fit, was imposed throughout the simulation. Figure 10 depicts an $\chi^{2}$ distribution in the inclination. There is a clear indication that the inclination is very close to $90^{\circ}$. Once this was established with confidence, the inclination was kept constant throughout subsequent analysis. The limb-darkening coefficients for the square-root law were automatically interpolated from van Hamme (1993) tables after each iteration.

It is obvious that neither the existing spectroscopy nor photometry allows us to derive the exact value of the contribution of the third light in the three photometric passbands. Guided by the finding that the spectral types of components Aa and Ba seem to
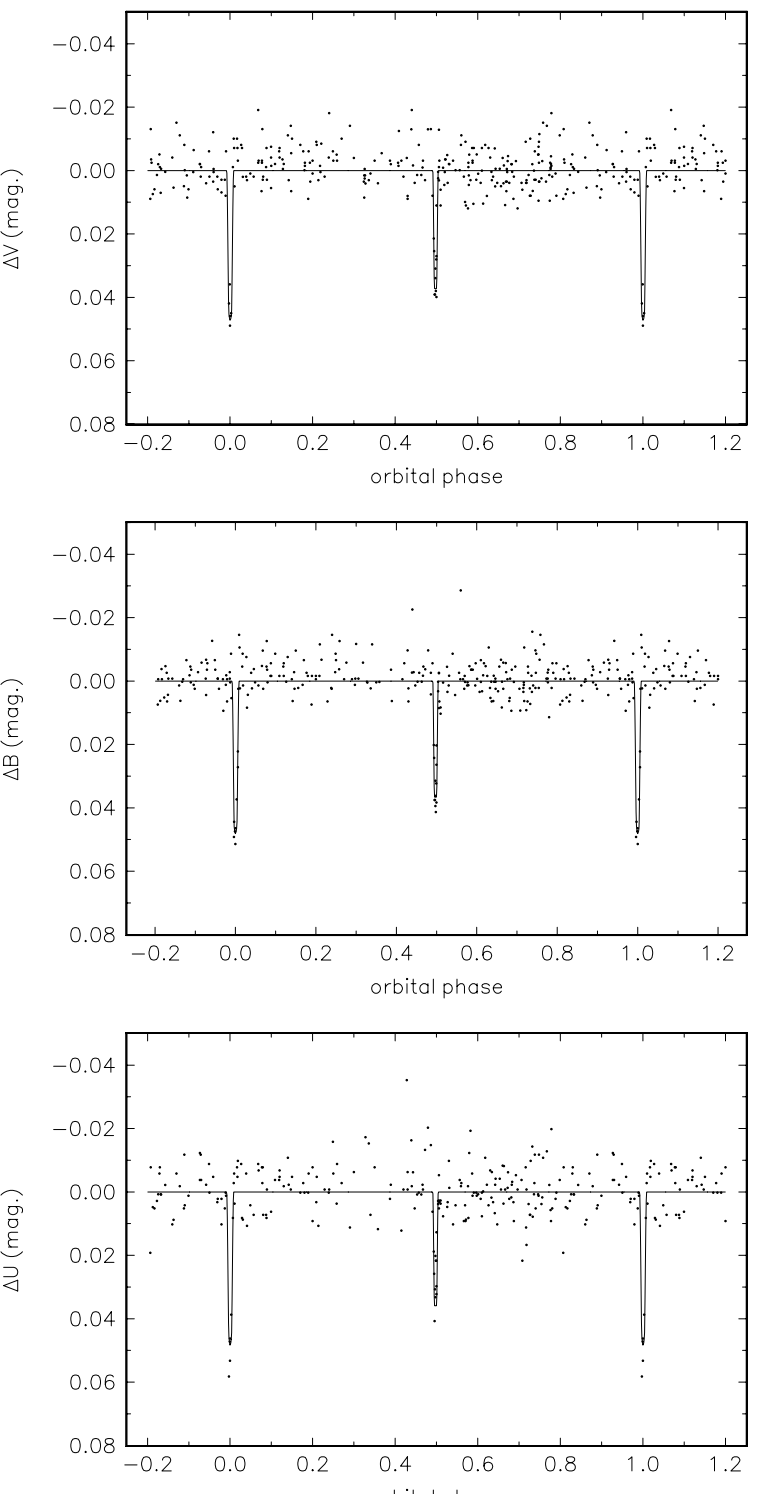

Fig. 11. The $V, B$, and $U$ light curves of the eclipsing system $\mathrm{Aa}+\mathrm{Ab}$ with the solution for a 70 per cent contribution of the light from B binary.

be nearly identical over the whole optical spectrum and by the luminosity ratio estimated from the disentangled line profiles, we derived several trial solutions in which we kept the contribution of the third light constant in all passbands and set to various values between 50 and 75 per cent of the total luminosity $\left(L_{1}+L_{2}+L_{3}\right)$ of the system.

It turned out that the most consistent results were obtained for a solution in which the third light represents 70 per cent of the total flux in all three passbands. Because of a distinct, widely detached configuration of both components, and the system's pronounced eclipses, we were able to put together a reliable model with the principal parameters listed in Table 7 . Figure 11 is the comparison of the complete observed and synthetic light curve in the $U B V$ passbands, while Fig. 12 shows a detail of the fit in both minima for the more numerous $V$ and $B$ data. Note that all these plots are constructed in such a way that the mean light level, derived individually for each passband and each dataset, was subtracted from both, the observed data and the model light curve. The corresponding RV curves are shown in Fig. 13. To 
Table 7. Solutions of the light and RV curves for the Aa $+\mathrm{Ab}$ binary derived with the PHOEBE program and assuming that the third light contributes 70 per cent in all three $U B V$ passbands; therefore $L_{2}=1-L_{1}-0.70$. The rms errors of the elements are formal errors, deduced from a covariance matrix. Values of the ratio of the orbital to the rotational period $F$, the albedo $A$, and the coefficients of the gravity darkening $\beta$ were all kept fixed in the solution.

\begin{tabular}{|c|c|c|c|}
\hline \multirow[t]{2}{*}{ Parameter: } & \multicolumn{3}{|c|}{ System: } \\
\hline & Primary: & & Secondary: \\
\hline$P(\mathrm{~d})$ & & $99.7638 \pm 0.0006$ & \\
\hline$T_{\text {periastr. }}$ & & HJD $2441905.585 \pm 0.046$ & \\
\hline$T_{\text {min.I }}$ & & HJD 2441856.285 & \\
\hline$T_{\min . I I}$ & & HJD 2441905.948 & \\
\hline$a\left(R_{\odot}\right)$ & & $231.0 \pm 1.5$ & \\
\hline$i\left(^{\circ}\right)$ & & $90.0 \pm 0.76$ & \\
\hline$q=M_{2} / M_{1}$ & & $0.576 \pm 0.006$ & \\
\hline$e$ & & $0.117 \pm 0.005$ & \\
\hline$\omega\left(^{\circ}\right)$ & & $268.32 \pm 0.52$ & \\
\hline$v_{\gamma}\left(\mathrm{km} \mathrm{s}^{-1}\right)$ & & $-20.20 \pm 0.22$ & \\
\hline$L_{3} /\left(L_{1}+L_{2}+L_{3}\right)$ & & 0.7 fixed & \\
\hline$T_{\mathrm{eff}}[\mathrm{K}]$ & $22025 \pm 428$ & & $20206 \pm 374$ \\
\hline$\Omega$ & $29.79 \pm 0.39$ & & $45.06 \pm 0.88$ \\
\hline$r=R / a$ & $0.0342 \pm 0.0005$ & & $0.0132 \pm 0.0002$ \\
\hline $\log g / g_{0}[\mathrm{cgs}]$ & $3.69 \pm 0.14$ & & $4.28 \pm 0.14$ \\
\hline$L_{U}$ & $0.267 \pm 0.001$ & & $0.033 \pm 0.001$ \\
\hline$L_{B}$ & $0.267 \pm 0.001$ & & $0.033 \pm 0.001$ \\
\hline$L_{V}$ & $0.266 \pm 0.001$ & & $0.034 \pm 0.001$ \\
\hline$F$ & 4.0 fixed & & 1.0 fixed \\
\hline$A$ & 0.6 fixed & & 0.6 fixed \\
\hline$\beta$ & 1.0 fixed & & 1.0 fixed \\
\hline$M\left[M_{\odot}\right]$ & $10.56 \pm 0.23$ & & $6.09 \pm 0.13$ \\
\hline$R\left[R_{\odot}\right]$ & $7.909 \pm 0.12$ & & $3.04 \pm 0.04$ \\
\hline$L\left[L_{\odot}\right]$ & $13804 \pm 66$ & & $1445 \pm 30$ \\
\hline
\end{tabular}

derive a stable solution for the epoch, period, and longitude of periastron, we restricted the convergency in the later stages using only photometry from both eclipses.

A final remark: the value of $v \sin i$ of about $17 \mathrm{~km} \mathrm{~s}^{-1}$, which we derived from the disentangled line profiles of component Aa implies that this star rotates about 4 times faster than what would be expected for the spin-orbit synchronization. No such value could be derived for the weak $\mathrm{H} \alpha$ line of component $\mathrm{Ab}$. We therefore kept the ratio of the orbital to the rotational period $F$ fixed at values 4 and 1 for the primary and secondary in the solution but verified that using values of 1 and 1 or 4 and 4 has no detectable effect on the solution, which is not surprising, considering the small fractional radii of both stars.

Given the fact that $\mathrm{Aa}+\mathrm{Ab}$ is an edge-on system $\left(i \approx 90^{\circ}\right)$, we were able to derive the relative photometric radii (and hence the values of the effective potentials $\Omega_{1}$ and $\Omega_{2}$ ) to a sufficient degree of accuracy despite the fact that eclipse coverage is poor and out-of-eclipse parts of light curves are flat and featureless.

To determine the effective temperatures of individual stars, we used a colour-constraining technique described in Prša \& Zwitter (2006). We derived $E(B-V)=0$. 580 from the standard Hvar photometry of the whole system outside eclipses. This agrees well with an earlier determination $E(B-V)=0$ m.57 by Hiltner (1956). Using our refined value, we obtain the dereddened colour index $(B-V)_{0}=-0$. 242 . This implies a mean effective temperature of the binary $T_{\text {eff }}=21816 \mathrm{~K}$, yielding the effective temperatures of the components to be $T_{\text {eff, } 1}=22025 \mathrm{~K}$ and $T_{\text {eff, } 2}=20206 \mathrm{~K}$.

Although our solution is internally consistent, we again recall that it depends on the adopted value of the third light in the system. Unfortunately, with the data at hand (poor eclipse coverage and shallow eclipses, therefore a modest $\mathrm{S} / \mathrm{N}$ ratio), it proved impossible to distinguish the third-light contribution reliably from all other contributions that have a similar influence on the light curves.

The ephemerides that we used to display the final RV (phase zero at periastron, ephemeris (2)) and light curves (phase zero at the primary minimum; ephemeris (3)) are based on the solution presented in Table 7 and read as follows:

$$
\begin{aligned}
T_{\text {periastr. }} & =\text { HJD } 2441905.585+99.7638 \times E, \\
T_{\min \text { I }} & =\text { HJD } 2441856.285+99.7638 \times E .
\end{aligned}
$$

We note that within the accuracy of data, ephemeris (2) does not differ significantly from the preliminary ephemeris (1).

\section{Radiative properties of components $\mathrm{Aa}, \mathrm{Ab}$, and $\mathrm{Ba}$}

Since the existing photometric observations of the eclipses of components $\mathrm{Aa}$ and $\mathrm{Ab}$ are still limited and do not cover the two minima completely, all our solutions and estimates must be considered preliminary.

However, some simple independent checks of the consistency of our results are still possible. In Fig. 14 we compare the positions of components $\mathrm{Aa}$ and $\mathrm{Ab}$, based on our final PHOEBE solution with the evolutionary tracks for stars between 5 and $12 M_{\odot}$ published by Schaller et al. (1992). Given all the uncertainties, the agreement with the derived component masses, 10.6 and $6.1 M_{\odot}$, is quite satisfactory. It seems to be well established that component $\mathrm{Aa}$ is already evolved, while component $\mathrm{Ab}$ is 

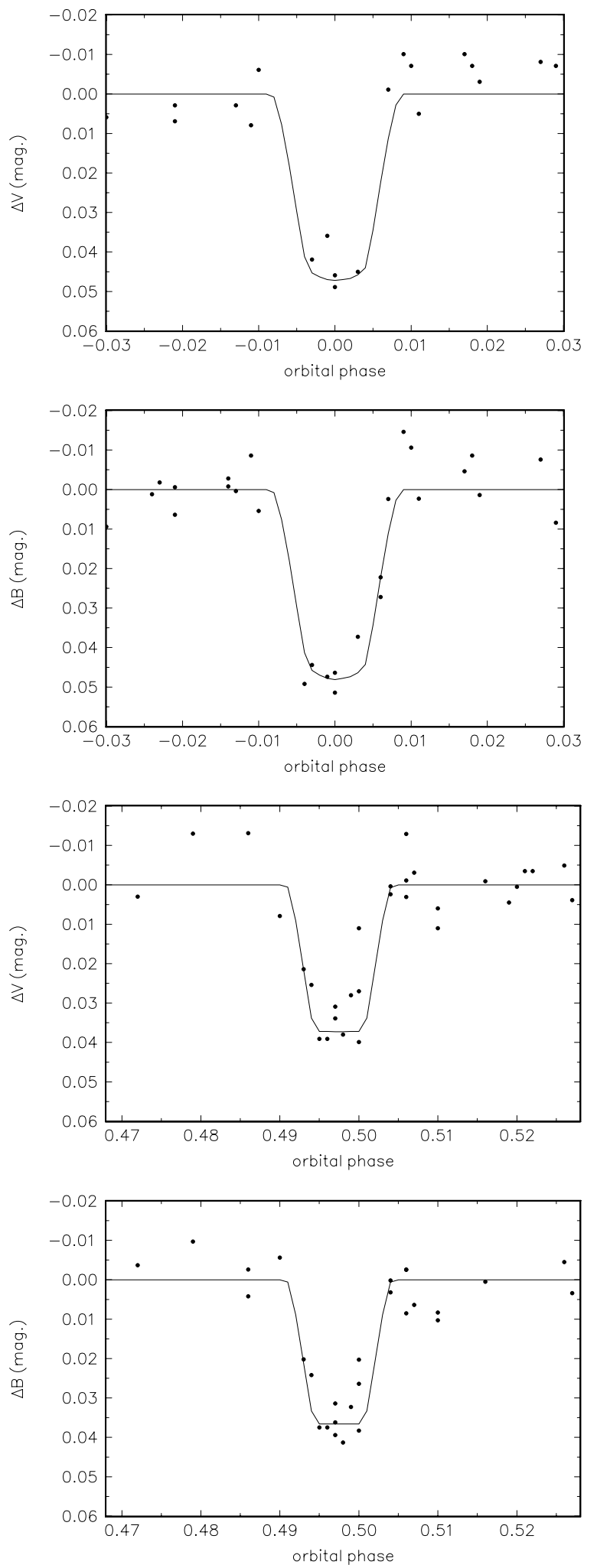

Fig. 12. The $V$ and $B$ light curves of the eclipsing system $\mathrm{Aa}+\mathrm{Ab}$ with the solution for a 70 per cent contribution of the light from B binary shown in the neighbourhood of both minima.

still very close to the zero-age main sequence. From the close similarity of the spectra of Aa and Ba over the whole optical range we conclude that the effective temperature of component $\mathrm{Ba}$ must also be close to $22000 \mathrm{~K}$.

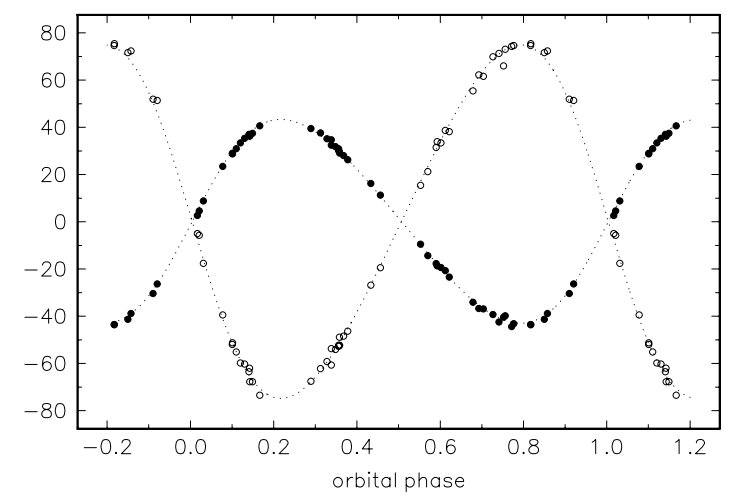

Fig. 13. Radial-velocity curves of the system $A a+A b$, based on KOREL disentangling and the final solution, plotted for ephemeris (2).

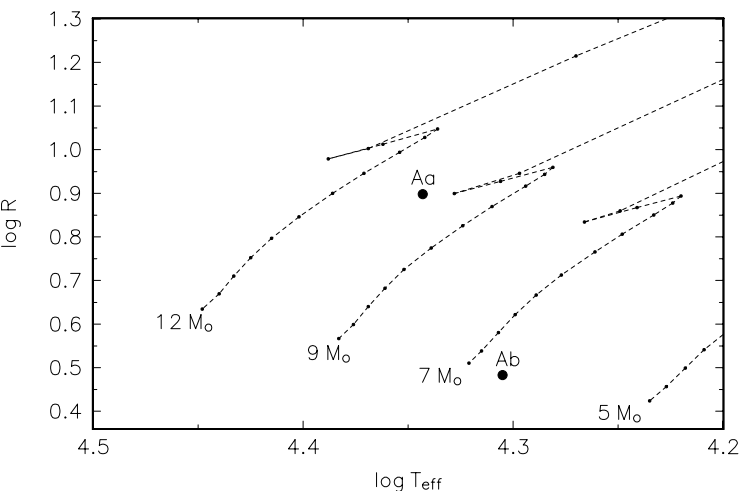

Fig. 14. The radii and effective temperatures of the components Aa and $\mathrm{Ab}$, based on the final PHOEBE solution, are compared with the evolutionary tracks for $12 M_{\odot}, 9 M_{\odot}, 7 M_{\odot}$, and $5 M_{\odot}$ models from Schaller et al. (1992).

\section{Probable physical properties of other bodies and the quadruple system}

If our solution for the $\mathrm{Aa}+\mathrm{Ab}$ binary is assumed valid, then the total mass of the A binary $M_{\mathrm{A}}=16.6 M_{\odot}$. After adopting $K_{\mathrm{A}}=13.2$ and $K_{\mathrm{B}}=20 \mathrm{~km} \mathrm{~s}^{-1}$ for the 7878-d orbit, $M_{\mathrm{B}}=$ 11.0 $M_{\odot}$. Then also $M_{\mathrm{A}} \sin ^{3} i_{\mathrm{AB}}=14.6 M_{\odot}$, i.e. $i_{\mathrm{AB}}=73^{\circ} 4$; and the semimajor axis is $5034 R_{\odot}$, i.e. $23.4 \mathrm{AU}$. The distance to V379 Cep might be about 500 ps, therefore the separation of binaries $A$ and $B$ might reach $\approx 50$ mas. At present, however, both binaries are close to a conjunction, therefore, with $i=73^{\circ} 4$, the angular separation would be under 20 mas. Mutual separations of the components $\mathrm{Aa}$ and $\mathrm{Ab}$, and $\mathrm{Ba}$ and $\mathrm{Bb}$ should be about 1 to 2 mas. Future attempts to resolve all these bodies via interferometry are very desirable.

For the B binary, we adopt the mean values of the three KOREL solutions from Table 6: $P=158.71 \pm 0.17, T_{\text {periastr. }}=$ HJD $2453004.48 \pm 0.27, e=0.509 \pm 0.016, \omega=59^{\circ} .4 \pm 1.8$, $K_{1}=15.60 \pm 0.71 \mathrm{~km} \mathrm{~s}^{-1}$. This implies $f(m)=0.0562 M_{\odot}$ and, since $M_{\mathrm{Ba}}+M_{\mathrm{Bb}}=11.0 M_{\odot}, M_{\mathrm{Bb}} \sin i_{\mathrm{B}}=1.90 M_{\odot}$. The mass of the Ba component should be close to Aa; i.e., the mass ratio in this orbit might be very small. This agrees with the fact that we were unable to disentangle any spectral lines of component $\mathrm{Bb}$. To visualize our model of the system, we provide Fig. 15 with a schematic diagram of it.

There is, however, still an unsolved problem in our model: Since the components Aa and Ba have very similar spectra, then the fact that the component Ba contributes some 70 per cent of the total light means that its radius must be about $13 R_{\odot}$, i.e. 


\section{V379 Cep}

Quadruple System

(not to scale)

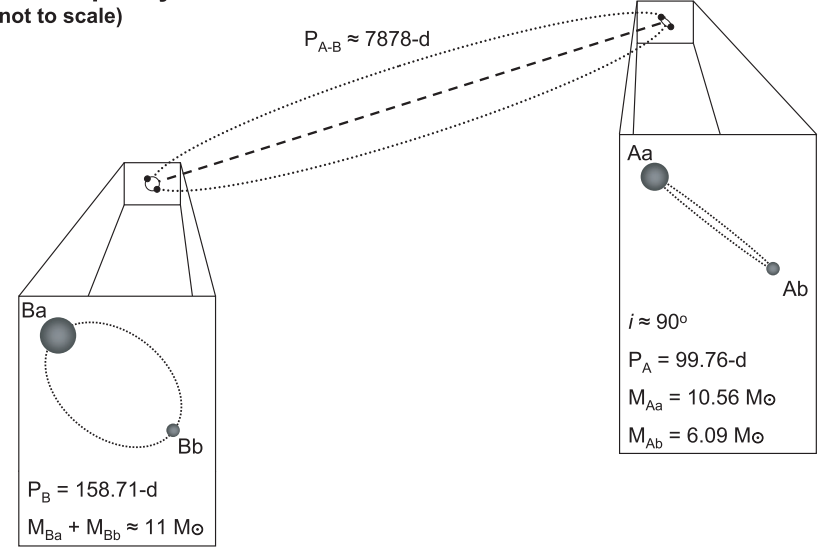

Fig. 15. A schematic visualization of our current model of the quadruple system.

larger than that of component Aa. At the same time, our current analysis implies that the mass of component $\mathrm{Ba}$ is slighly lower than that of component Aa, which should not be so in the case of a common origin of the system and its normal evolution. This could indicate either a real astrophysical problem or show that the remaining uncertainties in determining the properties of the four bodies are still large ones. The ultimate answer will only be possible after all three orbits are well-resolved and their inclinations derived.

Acknowledgements. We thank Drs. M. Jerzykiewicz and G. C. Clayton who provided us with their unpublished photometric observations. We acknowledge the use of the programs FOTEL, KOREL, and PREKOR, made available by their author, Dr. Petr Hadrava. This research was supported from the grants GA ČR 205/03/0788, GA ČR 205/06/0304, and GA CR 205/06/0584 of the Czech Science Foundation and also from the research project AV0Z10030501 of the Academy of Sciences of the Czech Republic. E. F. Guinan and G. McCook wish to acknowledge support from the US National Science Foundation Grant NSF/RUI AST-0507536. The mutual collaborative visits between Czech and Slovenian coauthors were facilitated thanks to project 22/2006-2007 of the Czech-Slovenian Intergovernmental Cooperation Programme. We acknowledge the use of the electronic bibliography maintained by NASA/ADS system and by the CDS in Strasbourg.

\section{References}

Abt, H. A. 1973, ApJS, 26, 365

Adams, W. S., Joy, A. H., \& Sanford, R. F. 1924, PASP, 36, 137

Clayton, G. C. 1996, PASP, 108, 401

Eggleton, P. P. 2002a, ApJ, 575, 1037

Eggleton, P. P. 2002b, in Exotic Stars as Challenges to Evolution, ASP Conf. Ser., 279, 37

Gordon, K. D., Clayton, G. C., Smith, T. L., et al. 1998, AJ, 115, 2561

Hadrava, P. 1990, Contributions of the Astronomical Observatory Skalnaté Pleso, 20, 23

Hadrava, P. 1995, A\&AS, 114, 393

Hadrava, P. 1997, A\&AS, 122, 581

Hadrava, P. 2004a, Publ. Astron. Inst. Acad. Sci. Czech Rep., 92, 1

Hadrava, P. 2004b, Publ. Astron. Inst. Acad. Sci. Czech Rep., 92, 15

Hadrava, P. 2005, Ap\&SS, 296, 239

Harmanec, P. 1998, A\&A, 335, 173

Harmanec, P., Horn, J., \& Juza, K. 1994, A\&AS, 104, 121

Harmanec, P., Pavlovski, K., Božić, H., et al. 1997, J. Astron. Data, 3, 5

Hiltner, W. A. 1956, ApJS, 2, 389

Horn, J., Kubát, J., Harmanec, P., et al. 1996, A\&A, 309, 521

Jerzykiewicz, M. 1993, A\&AS, 97, 421

Kaufer, A. 1988, Rev. Mod. Astrophys., 11, 177

Lynds, C. R. 1959, ApJ, 130, 577

Pavlovski, K., Harmanec, P., Božić, H., et al. 1997, A\&AS, 125, 75

Perryman, M. A. C., \& ESA. 1997, The HIPPARCOS and TYCHO catalogues, The Hipparcos and Tycho catalogues. Astrometric and photometric star catalogues derived from the ESA Hipparcos Space Astrometry Mission (Noordwijk, Netherlands: ESA Publications Division), ESA SP Series, 1200

Plaskett, J. S., \& Pearce, J. A. 1931, Publications of the Dominion Astrophysical Observatory Victoria, 5, 1

Prša, A., \& Zwitter, T. 2005, ApJ, 628, 426

Prša, A., \& Zwitter, T. 2006, Ap\&SS, 36

Schaller, G., Schaerer, D., Meynet, G., \& Maeder, A. 1992, A\&AS, 96, 269

Škoda, P. 1996, in Astronomical Data Analysis Software and Systems V, ASP Conf. Ser., 101, 187

Škoda, P., \& Šlechta, M. 2002, Publ. Astron. Inst. Acad. Sci. Czech Rep., 90, 40 van Hamme, W. 1993, AJ, 106, 2096

Wilson, R. E., \& Devinney, E. J. 1971, ApJ, 166, 605 